\title{
The cross-link breaker, $N$-phenacylthiazolium bromide prevents vascular advanced glycation end-product accumulation
}

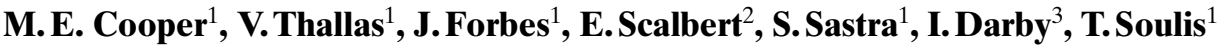 \\ ${ }^{1}$ Department of Medicine, University of Melbourne, Austin and Repatriation Medical Centre, West Heidelberg, Australia \\ ${ }^{2}$ IRIS, Courbevoie, France \\ ${ }^{3}$ Department of Human Biology, Royal Melbourne Institute of Technology, Bundoora Campus, Bundoora, Australia
}

\section{Abstract}

Aims/hypothesis. Advanced glycation is postulated to have a pivotal role in mediating diabetic vascular complications. The emergence of thiazolium compounds such as $\mathrm{N}$-phenacylthiazolium bromide which cleave preformed advanced glycation end products (AGEs) has allowed us to explore the effects of these agents on the vascular AGE accumulation and hypertrophy associated with diabetes.

Methods. Control and streptozotocin diabetic rats were selected at random for no treatment or treatment with $N$-phenacylthiazolium bromide $(10 \mathrm{mg} / \mathrm{kg}$ intraperitoneally) and followed for 3 weeks. In a separate study, intervention with $N$-phenacylthiazolium bromide was delayed until after 3 weeks of diabetes and then given for 3 weeks (total of 6 weeks).

Results. Diabetes was associated with increased mesenteric vascular advanced glycation end products, as assessed by radioimmunoassay and immunohistochemistry. This increase in vascular AGE accumulation was prevented by $\mathrm{N}$-phenacylthiazolium bromide treatment. Diabetes-associated mesenteric vascular hypertrophy was attenuated by treatment with $\mathrm{N}$-phenacylthiazolium bromide only if given from the time of induction of diabetes.

Conclusion/interpretation. Cross-link breakers seem to be effective in preventing or reversing accumulation of advanced glycation end-products in blood vessels and have the potential to play a part in the treatment of diabetic vascular complications. [Diabetologia (2000) 43: 660-664]

Keywords Glycation, vascular, thiazolium, cross-link breaker.
The accumulation of advanced glycated end products (AGEs), has been implicated in the pathogenesis of diabetic vascular complications [1]. Furthermore, an inhibitor of advanced glycation production formation, aminoguanidine has been shown to retard the progression of diabetic vascular complications [2].

Received: 28 September 1999 and in revised form: 10 February 2000

Corresponding author: M.E. Cooper MD, Department of Medicine, University of Melbourne, Austin and Repatriation Medical Centre, West Heidelberg, 3081 Australia

Abbreviations: AGE, Advanced glycation end product; PTB, $\mathrm{N}$-phenacylthiazolium; NO, nitric oxide; CML, carboxymethylysine; STZ, streptozotocin.
Because aminoguanidine has other actions including effects as an inhibitor of nitric oxide (NO) synthase [3], it is controversial whether the organ protective effects of aminoguanidine are solely due to its action as an inhibitor of AGE formation. The emergence of agents such as the thiazolium compounds [4] which promote the degradation of AGEs allows further exploration of the relative role of AGE accumulation in mediating vascular injury associated with diabetes. The efficacy of these agents in vivo in preventing AGE accumulation has not been extensively investigated. Our group has previously identified the mesenteric vascular tree as a site of AGE accumulation within 3 weeks of experimental diabetes [5]. This accumulation of AGEs has been linked to the development of mesenteric vascular hypertrophy which can 
be attenuated by agents which inhibit AGE formation, such as aminoguanidine and the more selective blocker, 2,3 diaminophenazine [6]. This study evaluated in vivo the effects of $N$-phenacylthiazolium bromide (PTB) on mesenteric vascular AGE accumulation and hypertrophy. Because it is hypothesised that PTB will cleave preformed cross-links in AGEs [4], the experimental protocol included a group of rats that received PTB as a delayed intervention commencing after 3 weeks of experimental diabetes and subsequently followed for 3 weeks of treatment.

\section{Materials and methods}

Male Sprague Dawley rats, aged 8 weeks, weighing between 200 and $250 \mathrm{~g}$ were selected at random to receive streptozotocin (STZ) at a dose of $45 \mathrm{mg} / \mathrm{kg}$ by intravenous injection after overnight fasting (diabetic) or citrate buffer alone (control). Animals were then further selected at random to receive either no treatment or PTB injected intraperitoneally at a dose of $10 \mathrm{mg} / \mathrm{kg}$ body weight on a daily basis (Study 1). In this study, animals were killed after 3 weeks of diabetes. In a separate group of control and diabetic rats, no treatment was commenced until 3 weeks after injection of citrate buffer or STZ (Study 2). These animals were selected at random after 3 weeks to receive treatment with PTB or no treatment for a further 3 weeks, resulting in a study duration of 6 weeks. All animals were given free access to standard chow containing $20 \%$ protein, (Clark, King, Melbourne, Australia). Only STZ-treated animals with plasma glucose concentrations greater than $15 \mathrm{mmol} / \mathrm{l} 1$ week after induction of diabetes were considered diabetic and included in the study. All diabetic animals received 4 units insulin/zinc suspension (Ultratard HM, Novo Nordisk, Bagsvaerd, Denmark) injected subcutaneously three times a week to maintain body weight and improve survival. Animals were killed after 3 weeks (Study 1) or 6 weeks (Study 2).

Immediately before killing, rats were weighed and their systolic blood pressure was determined by tail-cuff plethysmography [7]. Animals were killed by decapitation and blood collected for determination of glycated haemoglobin $\left(\mathrm{HbA}_{1 \mathrm{c}}\right)$ by HPLC (BioRad, Hercules, Calif., USA). The mesenteric vessels were then removed and stripped of surrounding fat, connective tissue and veins to yield the superior mesenteric arterial tree as described previously [2]. The vessels were weighed, frozen in liquid nitrogen and stored at $-80^{\circ} \mathrm{C}$ for subsequent assays of AGE concentrations.

Radioimmunoassay (RIA) for quantification of AGE in mesenteric vessels. Tissue homogenates from mesenteric vessels were obtained from a subset of animals and assayed for AGEs by RIA [8]. The antibody used in this assay was directed against advanced glycated bovine serum albumin (BSA). We used AGE-RNase for preparation of tracer and standards. The AGE-RNase was iodinated using lacto-peroxidase and the AGE-RNase standards were generated as a result of 6 months incubation of RNase with $0.5 \mathrm{~mol} / 1$ glucose in $0.2 \mathrm{~mol} / 1$ phosphate buffer, $\mathrm{pH} 7.2$ at $37^{\circ} \mathrm{C}$. Mesenteric vessel homogenates extracted with collagenase were incubated with the AGEBSA antibody and tracer for $5 \mathrm{~h}$ at room temperature [6]. Sheep anti-rabbit IgG was then added and incubated overnight at room temperature. Precipitation of proteins was carried out by addition of $8 \%$ polyethylene glycol 6000 . The minimum concentration of AGE-RNase detectable was $250 \mathrm{ng} / \mathrm{ml}$ pro- tein and the $\mathrm{CV}$ of this assay was $11.9 \%,(n=4)$ at a concentration of $2 \mu \mathrm{g}$ AGE-RNase/mg protein. The standard curve was generated with serial AGE-RNase dilutions. Radioactivity was measured using a gamma counter (Canberra Packard Instruments, St. Louis, Mo., USA) and the amount of AGEs present was expressed as equivalents of ng AGE-RNase.

Assessment of AGE concentrations in vessels by immunohistochemistry. To complement the results by RIA, localisation of AGE accumulation within tissues was assessed by an immunoperoxidase technique using the polyclonal anti-AGE antibody described previously which detects carboxymethylysine (CML) containing proteins but not pentosidine [8]. Formalinfixed sections $(4 \mu \mathrm{m})$ of mesenteric vessels embedded in paraffin were cut, placed on gelatinised slides, rehydrated and treated with $1 \% \mathrm{H}_{2} \mathrm{O}_{2}$ :methanol followed by incubation in protein blocking agent (Lipshaw-Immunon, Pittsburgh, Pa., USA) for $20 \mathrm{~min}$ at room temperature. Sections were then incubated with the anti-AGE antibody for $30 \mathrm{~min}$ at room temperature, washed in PBS and incubated with biotinylated swine antigoat, mouse, rabbit immunoglobulin (Dako, Carpinteria, Calif., USA) and peroxidase-conjugated streptavidin (Dako) [2]. Peroxidase conjugates were subsequently localised using diaminobenzidine tetrahydrochloride as a chromogen. To control for non-specific anti-AGE immunoreactivity, AGEs formed in vitro, as described above, were adsorbed against anti-AGE antibody and incubated in the normal staining procedure. Tissues treated in this manner showed no positive staining.

Statistics. All data are shown as means \pm SEM. Data were analysed by ANOVA using the Statview II program (Abacus Concepts, Berkeley, Calif., USA) on a Macintosh computer (Apple, Cupertino, Calif., USA). Comparisons between group means were analysed by Fisher's least significant difference method. A $p$ value less than 0.05 was considered statistically significant.

\section{Results}

Study 1 (Table 1). Diabetes was associated with reduced body weight and increased glycated haemoglobin values. Treatment with PTB did not affect body weight or glycaemic control. Diabetes was associated with a modest increase in systolic blood pressure which was attenuated by PTB therapy. In control rats, PTB treatment was associated with a tendency for blood pressure to increase. Diabetes was associated with a threefold increase in vascular AGE concentrations $(F=13.4, p<0.01$, Table 1$)$. Treatment with PTB prevented this increase in vascular AGE concentrations in the diabetic rats but did not affect these concentrations in control vessels $(F=12.9, p<0.01)$.

Immunohistochemical evaluation of mesenteric vessels suggested a similar pattern with a strong increase in AGE staining in the medial layer of diabetic vessels which was not evident in vessels from PTBtreated diabetic rats (Fig. 1). The increase in mesenteric vascular hypertrophy observed in the rats after 3 weeks of diabetes $(F=24.6, p<0.001)$ was attenuated but not restored to normal with PTB treatment $(F=6.5, p<0.015$, Table 1$)$. 
Table 1. Biochemical variables of Study 1 (early intervention)

\begin{tabular}{lcccc}
\hline Group & Control & Diabetic & Control + PTB & Diabetic + PTB \\
\hline$n$ & 9 & 7 & 8 & 6 \\
Final Body Wt (g) & $311 \pm 7$ & $272 \pm 8^{\mathrm{a}}$ & $351 \pm 10$ & $244 \pm 14^{\mathrm{a}}$ \\
$\mathrm{HbA}_{1 \mathrm{c}}(\%)$ & $4.4 \pm 0.1$ & $12.9 \pm 0.3^{\mathrm{a}}$ & $3.9 \pm 0.1$ & $11.6 \pm 1.0^{\mathrm{a}}$ \\
Blood Pressure (mm Hg) $_{\text {Mesenteric AGEs (ngRNase/ml) }}^{123 \pm 4}$ & $234 \pm 34$ & $140 \pm 6^{\mathrm{a}}$ & $145 \pm 3^{\mathrm{a}}$ & $126 \pm 4^{\mathrm{b}}$ \\
Mesenteric Weight (mg) & $30 \pm 1$ & $693 \pm 110^{\mathrm{a}}$ & $229 \pm 66$ & $157 \pm 83^{\mathrm{c}}$ \\
\hline
\end{tabular}

${ }^{\mathrm{a}} p<0.01$ vs control, ${ }^{\mathrm{b}} p<0.05,{ }^{\mathrm{c}} p<0.01$ vs diabetic

Table 2. Biochemical variables of Study 2 (delayed intervention)

\begin{tabular}{lcccc}
\hline Group & Control & Diabetic & Control + PTB & Diabetic + PTB \\
\hline$n$ & 10 & 7 & 9 & 9 \\
Body Wt $(\mathrm{g})$ & $400 \pm 8$ & $281 \pm 10^{\mathrm{a}}$ & $412 \pm 21$ & $276 \pm 13^{\mathrm{a}}$ \\
$\mathrm{HbA}_{1 \mathrm{c}}(\%)$ & $3.5 \pm 0.1$ & $14.7 \pm 0.5^{\mathrm{a}}$ & $3.5 \pm 0.1$ & $15.0 \pm 0.4^{\mathrm{a}}$ \\
Blood Pressure (mm Hg) $_{\text {Mesenteric AGEs }(\mathrm{ngRNase} / \mathrm{ml})}^{121 \pm 4}$ & $245 \pm 102$ & $139 \pm 5^{\mathrm{a}}$ & $135 \pm 6$ & $138 \pm 5^{\mathrm{a}}$ \\
Mesenteric Weight (mg) & $34 \pm 2$ & $1592 \pm 355^{\mathrm{a}}$ & $53 \pm 8$ & $227 \pm 108^{\mathrm{b}}$ \\
\hline
\end{tabular}

${ }^{\mathrm{a}} p<0.01$ vs control, ${ }^{\mathrm{b}} p<0.01$ vs diabetic

Study 2 (Table 2) In the delayed intervention study, diabetes was associated with reduced weight gain and increased glycated haemoglobin values (Table 2). Treatment with PTB did not influence body weight or glycaemic control. Diabetes was associated with a modest increase in blood pressure which was not affected by PTB treatment. Diabetes was associated with an approximate fivefold increase in vascular AGE concentrations $(F=20.4, p<0.001$, Table 2$)$. This increase in AGE concentrations was not seen in the group treated in a delayed manner for 3 weeks with PTB $(F=21.4, p<0.001)$. Furthermore, there was a tendency for delayed PTB treatment in control vessels to be associated with reduced AGE concentrations but this did not reach statistical significance $(p=0.07)$. Immunohistochemical evaluation showed a similar pattern with minimal detectable AGE immunostaining in control vessels or in vessels from PTB-treated control rats (Fig.1). By contrast, there was evidence of AGE immunostaining in the medial layer of diabetic vessels which was attenuated by delayed intervention with PTB. Diabetes was associated with mesenteric vascular hypertrophy $(F=86.9$, $p<0.001)$ but this variable was not influenced by delayed intervention with PTB (Table 2).

\section{Discussion}

Based on in vitro studies, it has been suggested that PTB is able to cleave preformed AGEs [4]. In our study, using RIA and immunohistochemistry it was possible to show that PTB treatment leads to reduced vascular AGE accumulation. The efficacy of this agent to reduce vascular AGE concentrations as part of a delayed intervention protocol is consistent with the postulated action of these thiazolium compounds to promote the degradation of preformed AGEs [4]. This was most clearly observed in the diabetic animals treated with PTB as was predictable because these animals were accumulating AGEs at a faster rate than control rats. An effect of this agent in the non-diabetic condition cannot, however, be excluded because AGEs accumulate over time in the absence of chronic hyperglycaemia. Note, in the delayed intervention study there was a trend for AGE accumulation to be reduced with PTB treatment in control rats. This emphasises the importance of exploring the role of cross-link breakers not only in diabetes.

Previous studies in our laboratory have characterised the AGEs which are detected by our immunoassay [8]. These studies suggest that the antibody detects predominantly, but not only, CML-containing proteins. It is likely that PTB cleaved AGEs detected by the RIA resulting in products which cannot be detected by the assay. Another possibility is that PTB treatment results in the generation of smaller AGEs which can then be more readily excreted in the urine. This concept that cross-link breakers could actually generate proteins which are preferentially excreted by the kidney has been suggested by a preliminary report which explored the effects of PTB in diabetic mice [9]. In that study, PTB treatment was associated with reduced renal AGE accumulation but the appearance of AGEs in the urine which had not been detected previously. The kidney and in particular the proximal tubule seems to be a major site of AGE clearance $[10,11]$. In the present study, PTB treatment was associated with attenuation of mesenteric vascular hypertrophy as a preventative strategy, as has been reported with aminoguanidine [2] and the 

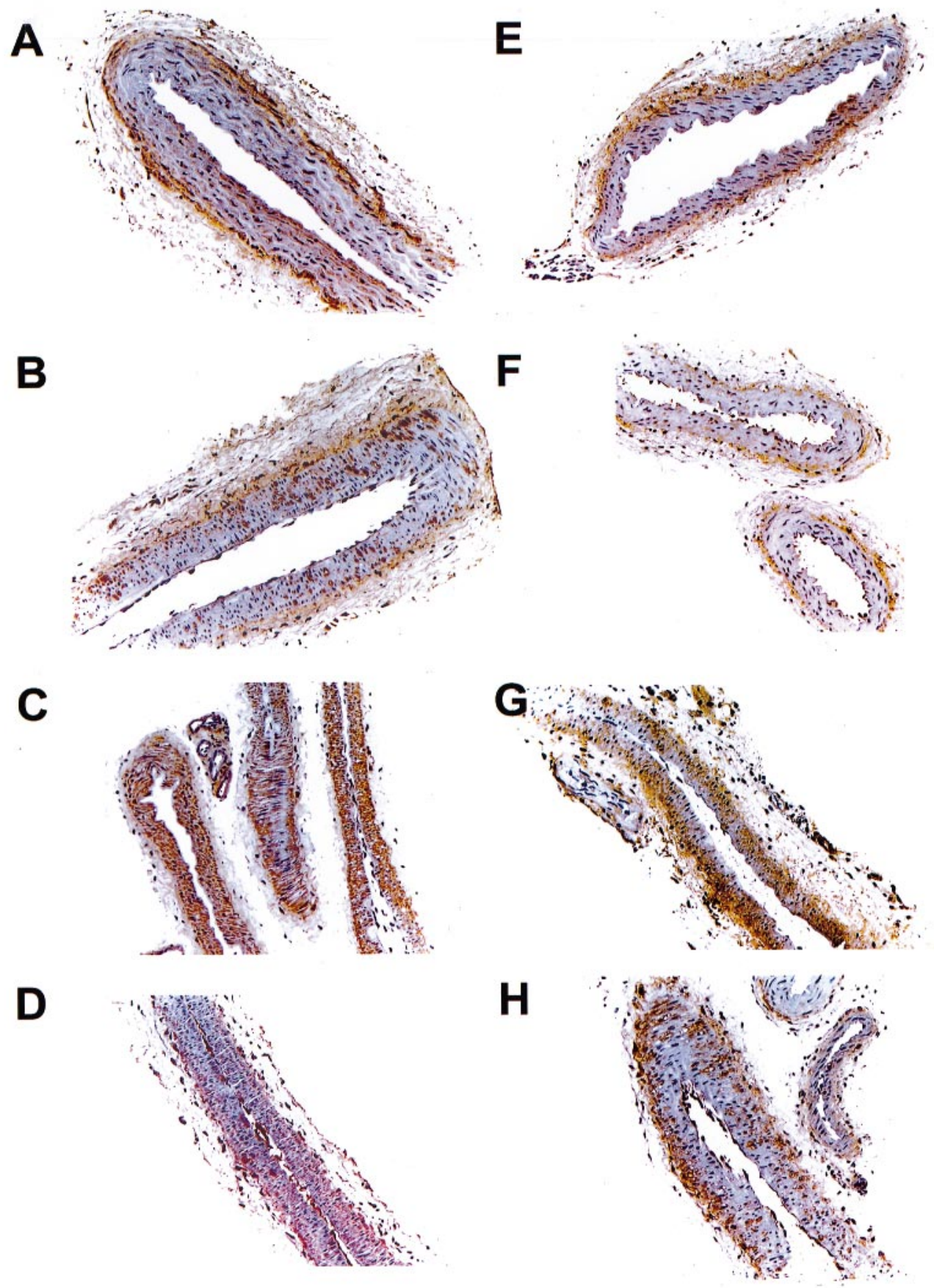

Fig. 1. Immunohistochemical staining for advanced glycation end-products in mesenteric blood vessels from animals in protocol 1 at 3 weeks: A control, B control + PTB, C diabetic, D diabetic + PTB and in protocol 2 at 6 weeks: $\mathbf{E}$ control, $\mathbf{F}$ control + PTB, $\mathbf{G}$ diabetic and $\mathbf{H}$ diabetic + PTB. Magnification $\times 400$

more selective inhibitor of AGE formation, 2,3 diaminophenazine [6]. These findings are consistent with our previous hypothesis that local vascular AGE accumulation is implicated in the development of vascular hypertrophy associated with diabetes [2]. The present study extends that concept by providing

evidence that vascular AGE accumulation can be controlled not only by agents which influence their formation but also by agents which promote their degradation.

This study provides in vivo evidence that the thiazolium class of compounds are able to retard AGE accumulation in vivo. The only other study which has explored the effects in vivo of cross-link breakers in diabetes used a stable derivative of PTB, ALT-711 [12]. In that study, ALT-711 was given for 1 or 3 weeks to rats after 9 weeks of streptozotocin diabetes. Treatment with ALT-711 reversed the decrease in large artery compliance that had been induced by diabetes. This was attributed to ALT-711 reversing the 
increase in collagen cross-linking, as assessed by an increase in susceptibility of collagen to be digested by pepsin. In that study vascular AGE accumulation was, however, not measured.

In the delayed intervention protocol, although PTB treatment reduced vascular AGE accumulation, no effect on the mesenteric vascular hypertrophy associated with diabetes was observed. It is possible that in the context of established AGE accumulation, 3 weeks of treatment was not long enough to reverse this process. It is likely that other factors are also involved in vascular hypertrophy at this site such as the local renin-angiotensin system as suggested by previous studies [13]. The findings of the present study contrast with those reported using ALT-711 where delayed intervention was effective in restoring vascular compliance in diabetic rats. Many factors could have caused the differences between the two studies such as the differences in the timing of intervention, the vascular variables assessed, the vascular site examined and the specific cross-link breaker used. Nevertheless, in both studies cross-link breakers were shown to influence vascular variables including vascular function and structure.

The mechanisms responsible for the various functional and structural changes in diabetic vessels have been increasingly explained over the last decade. Advanced glycation end products are viewed as quenching NO [14] and it is possible that the effect of PTB in attenuating the increase in blood pressure in the diabetic rats was related to reduced AGE accumulation leading to enhanced action of the vasodilator NO. By contrast, there seemed to be an unexplainable increase in blood pressure with PTB treatment in control rats. The matrix accumulation in diabetic vessels seems to be partly related to AGE [15] and involves overexpression of prosclerotic cytokines such as TGF $\beta$ with concomitant increased expression of matrix proteins [2]. It is still to be determined if the effects of aminoguanidine on these pathways are reproduced by cross-link breakers. If the major mechanism of action of aminoguanidine is by inhibition of AGE formation, it can be predicted that cross-link breakers could also play an important part in attenuating vascular disease associated with diabetes.

Acknowledgements. The authors wish to thank Dr D. Lesieur from the Albert Lespagnol Institute of Pharmaceutical Chemistry, Lille, France for synthesis of PTB. This study was supported by grants from Juvenile Diabetes Foundation International, the National Health and Medical Research Council of Australia and the National Heart Foundation of Australia.

\section{References}

1. Brownlee M (1994) Lilly Lecture 1993. Glycation and diabetic complications. Diabetes 43: 836-841

2. Rumble JR, Cooper ME, Soulis Tet al. (1997) Vascular hypertrophy in experimental diabetes: role of advanced glycation end products. J Clin Invest 99: 1016-1027

3. Corbett JA, Tilton RG, Chang K et al. (1992) Aminoguanidine, a novel inhibitor of nitric oxide formation, prevents diabetic vascular dysfunction. Diabetes 41: 552-556

4. Vasan S, Zhang X, Zhang X et al. (1996) An agent cleaving glucose-derived protein crosslinks in vitro and in vivo. Nature 382: 275-278

5. Cooper M, Rumble J, Komers R, Du H, Jandeleit K, Chou $S$ (1994) Diabetes-associated mesenteric vascular hypertrophy is attenuated by angiotensin-converting enzyme inhibition. Diabetes 43: 1221-1228

6. Soulis T, Sastra S, Thallas V et al. (1999) A novel inhibitor of advanced glycation end-product formation inhibits mesenteric vascular hypertrophy in experimental diabetes. Diabetologia 42: 472-479

7. Bunag R (1973) Validation in awake rats of a tail-cuff method for measuring systolic pressure. J Appl Physiol 34: 279-282

8. Soulis T, Cooper ME, Sastra S et al. (1997) Relative contributions of advanced glycation and nitric oxide synthase inhibition to aminoguanidine-mediated renoprotection in diabetic rats. Diabetologia 40: 1141-1151

9. Schwedler S, Verbeke P, Bakala H et al. (1998) N-Phenacylthiazolium bromide (PTB) decreases renal AGE levels and increases urinary AGE excretion, without ameliorating diabetic nephropathy in C57BL/6 mice. J Am Soc Nephrol 9: 641A (Abstract)

10. Youssef S, Nguyen DT, Soulis T, Panagiotopoulos S, Jerums G, Cooper ME (1999) Effect of diabetes and aminoguanidine therapy on renal advanced glycation end-product binding. Kidney Int 55: 907-916

11. Gugliucci A, Bendayan M (1996) Renal fate of circulating advanced glycated end products (AGE): evidence for reabsorption and catabolism of AGE-peptides by renal proximal tubular cells. Diabetologia 39: 149-160

12. Wolffenbuttel BHR, Boulanger CM, Crijns FRL et al. (1998) Breakers of advanced glycation end products restore large artery properties in experimental diabetes. Proc Natl Acad Sci USA 95: 4630-4634

13. Rumble JR, Gilbert RE, Cox A, Wu L, Cooper ME (1998) Angiotensin converting enzyme inhibition reduces the expression of transforming growth factor-beta(1) and type IV collagen in diabetic vasculopathy. J Hypertens 16: 1603-1609

14. Hogan M, Cerami A, Bucala R (1992) Advanced glycosylation endproducts block the antiproliferative effect of nitric oxide. Role in the vascular and renal complications of diabetes mellitus. J Clin Invest 90: 1110-1115

15. Huijberts MS, Wolffenbuttel BH, Boudier HA et al. (1993) Aminoguanidine treatment increases elasticity and decreases fluid filtration of large arteries from diabetic rats. J Clin Invest 92: 1407-1411 